State of the art in the traditional knowledge of the wild mushrooms in the Tlaxcala state, Mexico

\title{
Estado del arte relativo al conocimiento tradicional de los hongos silvestres en el estado de Tlaxcala, México
}

Laura Guideony Caamal-Caamal ${ }^{1}$, Adriana Montoya ${ }^{1,2 *}$, Laura Trejo-Hernández ${ }^{3}$, Citlalli Castillo- Guevara ${ }^{1,2}$.

${ }^{1}$ Maestría en Biotecnología y Manejo de Recursos Naturales, Universidad Autónoma de Tlaxcala, Ixtacuixtla, Tlaxcala, México.

${ }^{2}$ Centro de Investigación en Ciencias Biológicas, Universidad Autónoma de Tlaxcala, Km 10.5 Autopista San Martín Texmelucan-Tlaxcala, 90120. Ixtacuixtla, Tlaxcala, México.

${ }^{3}$ Laboratorio Regional de Biodiversidad y Cultivo de Tejidos Vegetales, Instituto de Biología, UNAM, sede Tlaxcala. Contiguo F.E.S. Zaragoza Campus III, Ex Fábrica San Manuel de Marcom sn, San Miguel Contla, Municipio de Santa Cruz, Tlaxcala, C.P. 90640, Tlaxcala, México.*Email:laura_guide10@hotmail.com https://doi.org/10.29267/mxjb.2017.2.1.1

\section{RESUMEN}

El objetivo de este artículo es resumir la información existente a la fecha, sobre el conocimiento tradicional y uso de los hongos silvestres en varias comunidades de Tlaxcala, México. Hay registros de la importancia cultural, alimentaria, medicinal, económica, y sobre la problemática que presentan los hongos tóxicos en algunas de estas comunidades, así como, de los beneficios que se obtienen por la venta de este recurso fúngico. La comercialización se ha destacado como una actividad que complementa el ingreso familiar, por lo que es considerada importante, al menos en algunas localidades. En estudios recientes se ha mostrado que tradicionalmente el uso de los hongos se preserva y está en proceso de cambio, se sugiere que el proceso de recolección se realiza de manera sostenible.

Palabras claves: Hongos comestibles, Importancia Cultural, Parque Nacional La Malinche, Comercialización.

\begin{abstract}
The objective of this article is to summarize the current existing information on the traditional knowledge and use of wild mushrooms in several communities in Tlaxcala, Mexico. There are records of the cultural, alimentary, medicinal, economic importance and the problematic that the toxic mushrooms present in some communities, as well as the benefits that are obtained by the sale of this fungal resource. Marketing has been highlighted as an activity that complements family income, so it has been considered an
\end{abstract}


important activity, at least in some localities. In recent studies it has been described as one of the most important resources for people, highlighting the ethnoecological knowledge that it has and because it is considered that the collection process is carried out in a sustainable way.

Keywords: Cultural importance, Edible fungi, La Malinche National Park, Marketing.

\section{INTRODUCCIÓN}

En México se han realizado estudios que describen la riqueza del conocimiento tradicional en torno a los hongos silvestres (Montoya et al., 2003). Estos son considerados como un recurso forestal no maderable de importancia alimentaria, para las comunidades rurales, ya que a partir de su recolección y comercialización éstas obtienen ingresos adicionales durante la temporada de lluvias y han contribuido en la conformación del conocimiento tradicional (Estrada-Martínez et al., 2009). El conocimiento micológico tradicional es descrito como el conjunto de conocimientos que las personas de las comunidades locales poseen sobre los hongos de su ambiente, dicho conocimiento es la base de todo el proceso de aprovechamiento de los hongos silvestres (Bautista, 2013). En el estado de Tlaxcala se han realizado estudios en varias comunidades principalmente en las aledañas al Parque Nacional la Malinche, sobretodo se ha escrito sobre el conocimiento tradicional, importancia cultural, categorías antropocéntricas, hongos tóxicos, clasificación tradicional y sobre las prácticas de manejo (Montoya et al., 2003).

El manejo se refiere a las transformaciones o decisiones sobre los ecosistemas naturales y artificiales, sus elementos (o recursos) y sus procesos funcionales (o servicios ecosistémicos) con fines explícitos. Son expresiones de manejo, las distintas formas de aprovechamiento (apropiación, obtención y uso de recursos, procesos y sistemas), las de conservación (acciones para mantenerlos y protegerlos) así como las guiadas hacia su restauración o recuperación (Casas et al., 2014). Los hongos son utilizados desde la época prehispánica, por lo que los diferentes grupos originarios y mestizos a lo largo del país y en otras partes del mundo, han desarrollado estrategias de uso y prácticas de manejo de las especies silvestres, procurando su propagación. Aunque no se ha medido el efecto de dichas prácticas para las especies micorrizógenas, es posible observar que los usuarios de los hongos, los cuidan y consideran que tales actividades están enfocadas en la conservación a futuro, debido a la importancia que representan desde el punto de vista cultural.

El conocimiento detallado de las estrategias de uso de los hongos y su relación con la importancia cultural son importantes aspectos que requieren caracterizarse, para entender la manera en que se realiza el manejo de estos organismos, los factores involucrados y determinar la importancia de los sitios de recolección en los bosques templados, lo que permitirá generar las bases para su conservación y uso sostenible.

\section{USO TRADICIONAL DE LOS HONGOS SILVESTRES}


Los hongos silvestres son organismos importantes constituyentes fundamentales de los ecosistemas, ya que intervienen activamente en los procesos de reciclaje de la materia orgánica e interacciones patogénicas o mutualistas con otros organismos como plantas, algas y animales (Montoya et al., 2003). Las comunidades rurales de diversas áreas poseen un profundo conocimiento tradicional sobre los hongos, además de un conocimiento biológico, por ejemplo, aspectos como sus estructuras morfológicas, origen, maneras de uso, forma de reproducción y conocimiento ecológico, pues se conoce en detalle el lugar en que fructifican y la asociación con algunos árboles, así como los aspectos sobre la época de fructificación de cada especie. El conocimiento etnomicológico es cada vez más estudiado y descrito, está centrado principalmente entre las comunidades campesinas e indígenas del país (Henao y Ruiz, 2006). Tienen gran importancia cultural; sin embargo, las especies más apreciadas varían de un grupo humano a otro dependiendo de las tradiciones, las formas de vida, los ambientes y ecosistemas y diversos factores (no suficientemente estudiados). Hasta ahora la información que la gente tiene, ha permitido el aprovechamiento y uso de los hongos con diferentes fines, entre ellos se tiene el uso alimentario, para venta, cosmético, como yesca, en la elaboración de artesanías, medicinal, insecticida y como "señal" (indicador, de la presencia de otros hongos, en tiempo y lugar). Otras categorías antropocéntricas detectadas son: tóxicos y enteógenos (esta última en el pasado).

\section{ESTUDIOS SOBRE EL CONOCIMIENTO TRADICIONAL DE LOS HONGOS SILVESTRES REALIZADOS EN TLAXCALA}

El Parque Nacional La Malinche (PNLM) o volcán La Malinche ("la de faldas azules"), se ubica en los territorios de los estados de Tlaxcala y Puebla, en la zona centro-oriente de México formando parte del Eje Volcánico Transmexicano y se considera la montaña aislada más significativa del país. Fue declarado como Parque Nacional mediante la publicación del Decreto en el Diario Oficial de la Federación el día 6 de octubre de 1938 (Torres-García, 2009). Se han realizado estudios etnomicológicos en diferentes comunidades, sobretodo en el sureste del estado, en aquellas que se ubican en las faldas del Parque en donde se encuentra una gran diversidad de especies de hongos silvestres (Figura 1). Con respecto al lugar de crecimiento de los hongos, la gente de la zona conoce cada región del bosque y las especies de hongos que pueden encontrar en cada una de ellas. De la misma manera, en las comunidades cercanas a los bosques de encino del centro de Tlaxcala, la gente reconoce varios lugares en los que se recolectan hongos (Montoya, 1997). 


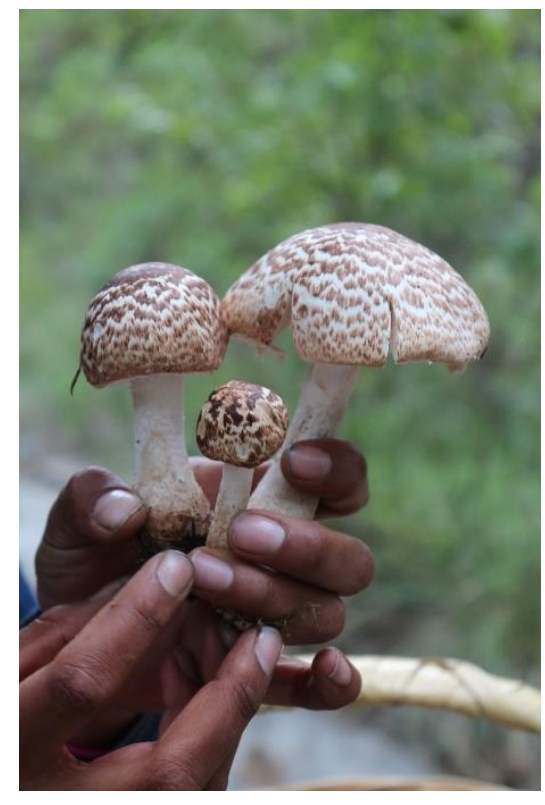

Fig. 1. Agaricus subrutilescens, hongo comestible, recolectado por los hongueros del Parque Nacional La Malinche, Tlaxcala.

Montoya (1989) realizó un estudio etnomicológico en tres comunidades de la región sureste; Ixtenco, Javier mina y los Pilares, en el que obtuvo datos sobre conceptos de hongos, nomenclatura, morfología, usos, fenología, ecología, clasificación tradicional, formas de preparación y criterios para identificar a las especies. En el trabajo se abordaron y discutieron aspectos sobre los métodos empleados para la obtención de la información. Una contribución metodológica de este trabajo es la realización de la comparación del conocimiento tradicional en comunidades con diferentes características, incluso diferente origen étnico y que comparten un mismo territorio con las mismas características de vegetación (PNLM), de donde obtienen diversos recursos naturales.

Montoya (1997) y Montoya et al. (2004) realizaron un estudio para describir los conocimientos locales sobre los hongos, de las personas de San Francisco Temetzontla, en la región Suroeste del estado, ubicada en la región geográfica conocida como Bloque Tlaxcala. Zona dominada por bosques de Quercus spp. y Pinus spp. El conocimiento de los hongos es relevante porque son utilizados como alimento pero también se desarrollan hongos potencialmente mortales que son conocidos y distinguidos por la gente. Los principales usos de los hongos en la comunidad son alimento y venta. Los hongos más apreciados son, el venadito (Amanita spp.), el hongo de llano (Agaricus campestris), los xoletes (Mariasmius oreades), las escobetas (Ramaria persicina y Ramaria spp.), las panzas (Boletus biiripes), los hongos blancos (Russula delica y $R$. aff. romagnesiana), el hongo de maíz (Ustilago maydis) y los rojos o colorados (Russula spp. secc. Insidiosae y Polychromae. El único hongo con fines medicinales es el "cuitlacoche" (Ustilago maydis), el cual se utiliza para curar quemadas o refrescar la piel. Resalta esta entidad por la diversidad de especies del género Russula que conocen y emplean en la alimentación. Con respecto a la venta es poco frecuente y pocas personas se dedican a la comercialización de 
hongos y en los más de los casos es local (7.1\%). Todos los habitantes de la comunidad señalan como venenosos a Boletus luridiformis y a Suillus glandulosipes, debido a que cambian de color, tornándose azules al corte, las personas consideran venenosos a todos aquellos que no conocen. Uno de los graves problemas es la necesidad económica de las personas además del incremento en el tamaño de la población han provocado la ampliación de las zonas dedicadas al cultivo de maíz, frijol y haba, afectando el tamaño del área forestal (Zenteno, 2007). En este trabajo también se abordaron aspectos metodológicos que se enfocan en la medición de la Importancia Cultural con el uso de un indicador denominado Valor de uso. Una contribución relevante a partir de este estudio es la descripción de una nueva especie de hongo para la ciencia, que es Russula herrerae = hongo blanco que es comestible e la zona. Este trabajo contribuye con el conocimiento de los hongos de los bosques de encino de Tlaxcala e incluye un listado comentado, 54 taxa fueron determinados a nivel específico.

En 1998 se dio a conocer un resumen de los conocimientos obtenidos hasta esa fecha en Tlaxcala, sobre diferentes aspectos etnomicológicos. El trabajo incluye un listado de 73 especies de hongos silvestres comestibles, con sus nombres tradicionales e información sobre hongos considerados venenosos (Montoya et al. 1998).

Montoya et al. (2000) realizaron una investigación con la finalidad de difundir y conservar el acervo culinario relacionado con los hongos silvestres, las recetas para la preparación de los platillos se obtuvieron a través de entrevistas con los pobladores de distintas regiones de Tlaxcala. Incluye 20 especies de hongos.

Se han realizado algunos estudios en los mercados de Tlaxcala, Apizaco y Huamantla, durante la época de lluvias, con la finalidad de monitorear la venta de hongos silvestres (Montoya et al., 2001), se han registrado los nombres tradicionales asignados a las especies, los precios de venta y la procedencia de las vendedoras. También, de manera particular, en la comunidad de Javier Mina la comercialización de hongos ha permitido que los hongos locales se distribuyan en otras regiones del país e incluso a otros países, Montoya et al. (2008) describieron el proceso de comercialización de hongos en dicha comunidad, incluyendo precios de venta; refieren un estudio de caso como ejemplo de este proceso, en una tienda local.

Montoya et al. (2003) realizaron un estudio en una localidad de San Isidro Buensuceso en el que describió el conocimiento tradicional en relación con los usos, se determinó la importancia cultural con la técnica de listado libre. A través de dos indicadores que son frecuencia y orden de mención se obtuvieron valores para los hongos más importantes desde el punto de vista cultural, estos son: Turbinellus floccocosus, Boletus atkinsonii, Boletus pinophilus y Ramaria spp. Los hongos comestibles para la comunidad representan una fuente alimentaria muy importante y con potencial económico medicinal e insecticida. El uso más importante para la comunidad fue el autoconsumo (alimento). Las personas mencionan 11 nombres nahua para hongos tóxicos, solo indicaron algunos hongos en español, los conocen como "hongos malo" o "venenosos". Los nombres nahua asignados son de gran importancia para los habitantes de San Isidro Buensuceso, debido a en ellos representan las diferencias entre hongos comestibles y de los hongos tóxicos. Cabe 
mencionar que en San Isidro Buensuceso se registraron 48 especies. Este trabajo representa una contribución a la descripción del conocimiento Nahua de los hongos en la entidad.

Montoya (2005) describe algunos aspectos sobre la importancia cultural de los hongos y los factores que están relacionados ésta, en particular aspectos ecológicos como producción, abundancia y riqueza en el bosque, además de la comercialización. Es un trabajo que permite regionalizar al PNLM, en dos zonas, la ladera sureste, predominante mestiza, con una producción natural de pocas especies con mayor abundancia, comparada con la región suroeste, en la que hay menor abundancia de muchas especies y es un área mayormente indígena, por la presencia en varias comunidades del grupo originario nahua.

Aunque la mayoría de los estudios Etnomicológicos en Tlaxcala se han realizado en el Parque Nacional La Malinche, algunos, se han realizado en otras regiones. Al Norte del Estado, Zenteno (2007) describió el conocimiento general de los hongos silvestres entre los habitantes de San Felipe Hidalgo, municipio de Nanacamilpa. En esta localidad la mayoría de las personas hacen un aprovechamiento de estos organismos, ya sea para autoconsumo o venta, establecen parámetros que permiten identificar si un hongo es comestible o tóxico (color, olor y textura). Esta actividad les permite seguir transmitiendo sus conocimientos a las nuevas generaciones, permite la convivencia entre los integrantes de la comunidad. En la población hay una zona donde se encuentran una gran variedad de hongos, pero sólo se consumen los que han sido recolectados por las personas de generaciones pasadas (adultos). Entre los hongos más apreciados están los siguientes, el xolete, tecomate, escobetas, pananacas, tecosita, cornetas y elotes, entre otros. Sólo esperan la temporada de lluvias para aprovechar este recurso. Para el manejo y extracción de los hongos consideran las sugerencias de sus antepasados, por lo que no cortan el micelio debido a que si lo hacen, no habrá hongos el próximo año. Se realiza la comercialización de los hongos en gran escala ya que los distribuyen a la Ciudad de México, San Martín Texmelucan (Puebla), Calpulalpan, Nanacamilpa (Tlaxcala) y Estado de México.

Reyes-López (2007) realizó un estudio sobre clasificación nahua de hongos silvestres en la comunidad de San Isidro Buensuceso, encontrando que la clasificación que hicieron las personas obedece a criterios pragmáticos. Sugiere la existencia de varias maneras de clasificar a los hongos con el uso de distintos criterios, unas son más evidentes que otras. En este trabajo se abordó el concepto de hongo utilizando la técnica de triadas.

Montoya et al. (2009) realizaron un estudio donde describen el conocimiento tradicional de los hongos silvestres (lugar, y época de crecimiento, usos, formas de preparación y nombre asignados a las estructuras fúngicas) en la comunidad de Altamira de Guadalupe, Huamantla, toda la información se obtuvo por medio de entrevistas en el lugar y se realizaron recorridos a los bosques del volcán la Malinche. Los hongos más importantes para esa comunidad fueron; pante (Boletus pinophilus), jícara amarilla (Amanita bassi) xolete (Lyophillum decastes) y tecosita (Cantharellus cibarius). De manera anual, realizan una demostración gastronómica basada en hongos para la clausura del ciclo escolar, de este modo, se evidencia que este recurso es de importancia para la zona. Los principales usos de los hogos en la zona son el alimentario y la venta. De estos resalta el alimentario pues se 
tienen varias formas de preparación de los diferentes hongos en la comunidad. En cuanto a hongos tóxicos el más conocido es la jícara roja (Amanita muscaria), es claro que la gente tiene mucha experiencia y no recolectan, no se comen, ni venden los hongos que presentan un riesgo a la salud. Esta actividad ha permitido conservar una tradición de mucha antigüedad. Por lo que este conocimiento se va transmitiendo de padres a hijos y se enriquece por la interacción con otras comunidades. El conocimiento descrito en este trabajo concuerda con los reportados para otras comunidades aledañas al volcán la Malinche. En este trabajo es importante resaltar el hecho de la importancia alimentaria de los hongos silvestres, la realización de una muestra gastronómica de hongos cada año, es una evidencia en este sentido y caracteriza a la comunidad.

Torres-García (2009) evaluó la importancia cultural de los hongos en 10 comunidades: San Isidro Buensuceso, Acxotla del Monte, San Francisco Tetlanohcan, San Rafael Tepatlaxco, Ixtenco, Francisco Javier Mina, Altamira de Guadalupe, Pueblo de la Cruz, Pueblo de Mariano Matamoros y en los Pilares. Entre las comunidades hay algunas mestizas y otros grupos originarios como el nahua y otomí. El uso alimentario y la venta son una gran tradición para las comunidades, además, son los principales en utilizar este recurso con fines económicos, es decir la comercialización en los mercados locales. Considerando la frecuencia de mención de los hongos, las especies más apreciadas e importantes en las comunidades, de acuerdo con los indicadores empleados son: Amanita bassi, Lyophyllum decastes, Boletus pinophilus, Turbinellus floccosus, Cantharellus gpo. cibarius, Russula delica, Ramaria sp. Lacaria trichodemophora, Suillus psuedobrevipes, Morchella aff. esculenta y Amanita muscaria. La contribución más importante de este trabajo, es la valoración de la importancia cultural de los hongos en 10 comunidades ubicadas en las faldas del Volcán La Malinche.

Alonso-Aguilar et al. (2011) estimaron la importancia cultural de los hongos silvestres en San Mateo, Huexoyucan. Se utilizó un índice de significancia cultural el cual incluye siete subíndices, que permitieron determinar las razones de la importancia para cada especie de hongos. El subíndice de medicina y el económico, no resultaron de utilidad en la comunidad estudiada, ya que todos utilizan el recurso como alimento y no para venta. Las especies con mayor importancia fueron: Amanita gpo. caesarea, Agaricus campestris, Russula spp., Ramaria spp. y Boletus variipes. Con respecto a frecuencia de mención los hongos con valores más altos fueron; Calvatia cyathiformis, Marasmius oreades, Agaricus campestris y Amanita gpo. caesarea. En cuanto a orden de mención Agaricus campestris fue la más importante. La contribución de este trabajo es desde el punto de vista metodológico, ya que se usa un índice para medir la importancia cultural de los hongos.

Vázquez-Núñez (2012) realizó un estudio en la comunidad de San Mateo Huexoyucan, en el que describe el conocimiento local sobre 42 especies de hongos silvestres. Las personas consideran que los hongos son un grupo diferente, distinto de plantas y animales (98.00\%), señalaron que "brotan de la tierra", que brotan solitos, que se producen en la temporada de lluvias y por ello son considerados organismos fríos. Con respecto a la información etnoecológica, se tiene un conocimiento detallado del lugar de crecimiento de cada especie. Los principales usos registrados son alimentario y venta. En el trabajo se incluyen recetas tradicionales obtenidas para su preparación. El principal aporte de este trabajo es el listado 
de las especies útiles en la zona y que proceden de los bosques de Quercus (encino) de Tlaxcala.

Entre otros aspectos analizados sobre los hongos silvestres, se ha evaluado el potencial antigenotóxico de Cantharellus cibarius el cual fue evaluado por Méndez-Espinoza et al. (2013). Este hongo fue recolectado en el Parque Nacional la Malinche y está entre las especies de mayor venta en algunos mercados y es apreciada como un buen comestible en varias comunidades ubicadas en las faldas del volcán. Se probaron las propiedades biológicas para conocer su potencial como agente antigenotóxico, como un tratamiento alternativo-quimiopreventivo. Los autores utilizaron células humanas mononucleares expuestas a metil-metanosulfonato, en diferentes extractos acuosos. Se empleó el ensayo cometa como método de análisis. Mostraron que el hongo presentó el más alto efecto inhibitorio en los tratamientos preventivo y simultáneo. Los autores discuten el papel nutrimental, ecológico y medicinal de este hongo (complejo de especies). Diferentes fuentes han mostrado sus propiedades antigenotóxicas ante diferentes compuestos, debido a los contenidos de ácido ascórbico; el potencial antioxidante, para prevenir el daño al DNA en los linfocitos de personas fumadoras, por la presencia de beta-carotenos.

Bautista-González (2013), al realizar un estudio sobre los hongos medicinales en seis estados del centro del país, incluye a San Isidro Buensuceso, Tlaxcala en su análisis y registra 36 especies con uso medicinal en la comunidad.

Ramírez-Terrazo (2017) investigó a través de un estudio etnomicológico la importancia y el conocimiento tradicional de los hongos tóxicos en dos comunidades ubicadas en las Faldas del volcán la Malinche. Describe las creencias en torno a este grupo de hongos, la forma en que se clasifican y da especial énfasis a los criterios de reconocimiento de los hongos tóxicos. Es un trabajo innovador en lo que respecta al tema estudiado.

La Tabla 1 muestra una comparación breve de algunos trabajos que dan una idea de las investigaciones realizadas y los temas estudiados, resaltando varios aspectos de interés.

\section{MÉTODOS ETNOGRÁFICOS Y BIOLÓGICOS EMPLEADOS EN LAS INVESTIGACIONES REALIZADAS}

El conocimiento tradicional en las comunidades es de suma importancia debido al grado de información que se tiene acerca de los hongos comestibles y los nombres comunes o tradicionales que aún se conservan. La comunidad de San Isidro Buensuceso fue donde se obtuvo un mayor conocimiento con los hongos silvestres en cuanto a nombres tradicionales (105) (Figura 2), seguido Javier Mina. La nomenclatura tradicional utilizada por las personas de la zona de estudio para las especies comestibles, es sobresaliente por la variedad de nombres encontrados. En las comunidades de Tlaxcala se hablan dos lenguas que son el Nahuatl y el Otomí. En cuanto a los hongos tóxicos y medicinales, los pobladores conocen parte de la gran variedad de especies que existen, sólo en una comunidad se menciona un alto número de especies de hongos tóxicos y para usos medicinales no fue popular para los pobladores, comparado con uso alimenticio (Tabla 1). 
Tabla 1. Resumen de la información contenida en algunos de los trabajos realizados en Tlaxcala, sobre el Conocimiento tradicional de los hongos silvestres (-Información no mencionada).

\begin{tabular}{|c|c|c|c|c|c|c|c|}
\hline & $\begin{array}{l}\text { Nombre } \\
\text { Común }\end{array}$ & $\begin{array}{l}\text { Hongos } \\
\text { Comestibles }\end{array}$ & $\begin{array}{l}\text { Hongos } \\
\text { Tóxicos }\end{array}$ & $\begin{array}{l}\text { Hongos } \\
\text { Medicinales }\end{array}$ & Usos & Idioma & Referencia \\
\hline Nanacamilpa & 22 & 18 & 4 & $\begin{array}{ll}- & 2\end{array}$ & & Español & $\begin{array}{l}\text { Zenteno, } \\
2007\end{array}$ \\
\hline Ixtenco & 70 & 25 & - & 4 & & Otomí & $\begin{array}{c}\text { Montoya, } \\
1989\end{array}$ \\
\hline Javier mina & 104 & 38 & - & 4 & & Nahua & $\begin{array}{c}\text { Montoya, } \\
1989\end{array}$ \\
\hline Los pilares & 52 & 25 & - & 1 & & Español & $\begin{array}{c}\text { Montoya, } \\
1989\end{array}$ \\
\hline $\begin{array}{l}\text { Altamira de } \\
\text { Guadalupe }\end{array}$ & 56 & 45 & 6 & - & & Español & $\begin{array}{l}\text { Montoya et } \\
\text { al., } 2009\end{array}$ \\
\hline $\begin{array}{l}\text { San Isidro } \\
\text { Buensuceso }\end{array}$ & 105 & 31 & 18 & 36 & & Nahua & $\begin{array}{l}\text { Montoya et } \\
\text { al., } 2003 \\
\text { Bautista, } \\
2013\end{array}$ \\
\hline $\begin{array}{l}\text { San Francisco } \\
\text { Temetzontla }\end{array}$ & 96 & 46 & 12 & 1 & & Español & $\begin{array}{l}\text { Montoya et } \\
\text { al.,1997; } \\
2004\end{array}$ \\
\hline $\begin{array}{l}\text { San Mateo } \\
\text { Huexoyucan }\end{array}$ & 96 & 97 & - & - & & Español & $\begin{array}{l}\text { Alonso- } \\
\text { Aguilar et } \\
\text { al., 2011; } \\
\text { Vázquez, } \\
2012\end{array}$ \\
\hline $\begin{array}{l}\text { Diez } \\
\text { Comunidades }\end{array}$ & 298 & 49 & - & - & & $\begin{array}{l}\text { Español } \\
\text { Otomí } \\
\text { Nahua }\end{array}$ & $\begin{array}{l}\text { Torres- } \\
\text { García, } \\
2009\end{array}$ \\
\hline
\end{tabular}




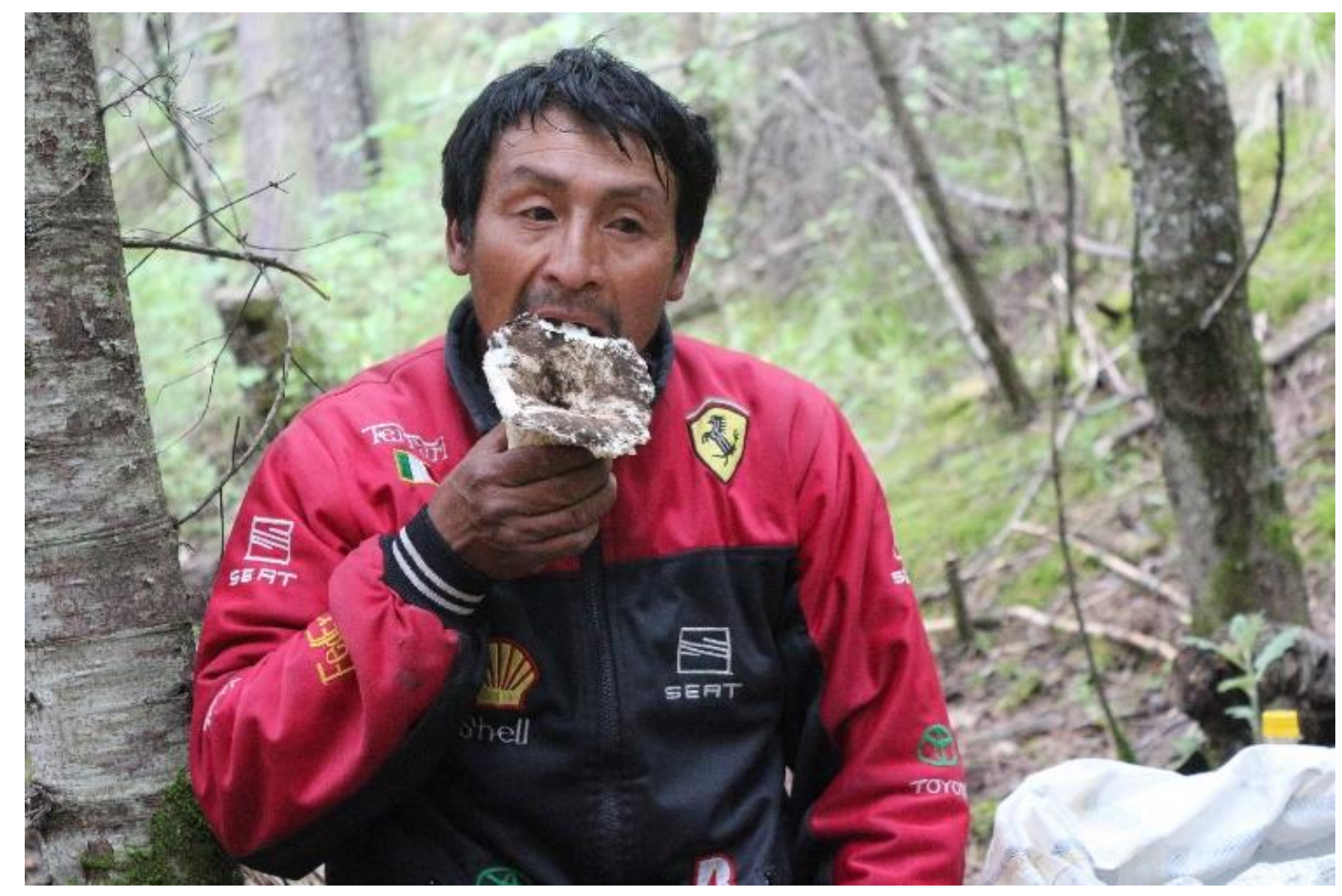

Fig. 2. Domingo Domínguez, recolector de hongos de la comunidad Nahua de San Isidro Buensuceso, Tlaxcala, probando un tecaxnanacatl (Russula delica).

Los métodos etnográficos utilizados para medir la importancia cultural de los hongos silvestres en todas las comunidades fueron fundamentales en las entrevistas y cuestionarios. Se observa que se han ensayado diferentes métodos como son el empleo de fotografías como estímulo; para la obtención de información, tanto entrevistas como cuestionarios; técnicas de triadas y sorteo de cartas (Tabla 2). Los estudios se han realizado en comunidades, en mercados, en centros hospitalarios, en el caso de hongos tóxicos y la zona más estudiada ha sido el Parque Nacional La Malinche. Otras regiones al norte y en el sureste han sido poco estudiadas. Los tipos de vegetación de los que se han obtenido los hongos y que están cercanos a las comunidades estudiadas son: bosques de coníferas y bosques de encino, aunque también se hace referencia a hongos que fructifican en zonas agrícolas como el cuitlacoche y el hongo de maguey. Falta aún la realización de estudios en otras áreas cuya cubierta de vegetación es aún considerable, como en la zona de Tlaxco, en Terrenate, Nanacamilpa y la zona oriente del estado de Tlaxcala. 
Tabla 2. Herramientas metodológicas utilizadas en los estudios realizados en algunas comunidades de Tlaxcala (*) Métodos empleados (-) Método no empleado).

\begin{tabular}{|c|c|c|c|c|c|}
\hline & Entrevistas & Cuestionarios & $\begin{array}{l}\text { Recorrido } \\
\text { Etnomicológicos }\end{array}$ & $\begin{array}{l}\text { Listado } \\
\text { libre }\end{array}$ & $\begin{array}{l}\text { Estímulo } \\
\text { fotográfico }\end{array}$ \\
\hline $\begin{array}{l}\text { Nanacamilpa } \\
\text { (San Felipe } \\
\text { Hidalgo) }\end{array}$ & 11 & 175 & $*$ & - & - \\
\hline Ixtenco & 92 & 390 & $*$ & $*$ & - \\
\hline Javier mina & 43 & 31 & $*$ & $*$ & - \\
\hline Los pilares & 56 & 74 & $*$ & $*$ & - \\
\hline $\begin{array}{l}\text { Altamira de } \\
\text { Guadalupe }\end{array}$ & 58 & & $*$ & $*$ & - \\
\hline $\begin{array}{l}\text { San Isidro } \\
\text { Buensuceso }\end{array}$ & 220 & 215 & $*$ & $*$ & $*$ \\
\hline $\begin{array}{l}\text { San } \\
\text { Francisco } \\
\text { Temetzontla }\end{array}$ & 68 & 215 & $*$ & $*$ & $*$ \\
\hline $\begin{array}{l}\text { San Mateo } \\
\text { Huexoyucan }\end{array}$ & 166 & - & $*$ & $*$ & - \\
\hline
\end{tabular}

\section{CONCLUSIONES}

La importancia de este trabajo es que resume las investigaciones llevadas a cabo sobre el conocimiento tradicional de hongos silvestres en diversas comunidades de Tlaxcala, en las que se describe una gran parte del conocimiento de los pobladores, así también permite visualizar estrategias para la conservación de este recurso y su manejo. 


\section{CONFLICTO DE INTERESES}

Los autores declaran que no existe conflicto de intereses.

\section{REFERENCIAS}

Alonso-Aguilar L E., Montoya A., Kong A., Estrada-Torres A., \& Garibay-Orijel R. 2014. The cultural significance of wild mushrooms in San Mateo Huexoyucan, Tlaxcala, Mexico. Journal of Ethnobiology and Ethnomedicine, 10, 27. http://doi.org/10.1186/17464269-10-27

Bautista-González J A. 2013. Conocimiento tradicional de hongos medicinales en seis localidades diferentes del país. Universidad Autónoma de México. Facultad de ciencias.

Casas A., Camou A., Otero-Arnaiz A., Rangel-Landa A. Cruse-Sanders J., Solís L., Torres H., Delgado A., Moreno-Calles A I., Vallejo M., Guillen S. BlancasJ., Parra F., FarfanHeredia B., Aguirre-Dugua X., Arrellanes Y., Perez-Negrón E. 2014. Manejo tradicional de biodiversidad y ecosistemas en Mesoamérica: el Valle de Tehuacán. Investigación ambiental 6(2).

Estrada-Martínez E., Guzmán G., Cibrián T. D., \& Ortega P. R. (2009). Contribución al conocimiento Etnomicológicos de los hongos silvestres comestibles en los mercados regionales y comunidades de la sierra nevada (México). Interciencia, 34(1), 25-33.

Henao L. y Ruiz A. 2006. Investigación y Gestión local de robledales alrededor del uso tradicional de macromicetos en la Cordillera Oriental Colombiana. En: Solano, C. y N. Vargas (ed.). Memorias del I Simposio de Robles y Ecosistemas Asociados. Bogotá. Fundación Natura y Pontificia Universidad Javeriana.

Méndez- Espinoza C., García-Nieto E., Montoya A., Montiel-Gonzáles A M., VelascoBautista E., Calderón-Ezquerro C., Juárez-Santacruz L.2013. Antigenotoxic Potential of Aqueous Extracts from the Chantarelle Mushroom, Chantarellus cibarius (Higher Basidiomycetes), on human mononuclear cell cultures. International Journal of Medicinal Mushrooms. 15(3): 325-332. DOI: 10.1615/ IntJMedMushr.v 15.i3.90.

Montoya A. 1989. Análisis comparativo de la Etnomicología de tres comunidades ubicadas en las faldas de la Malintzi, Estado de Tlaxcala. Tesis profesional, E.N.E.P., Iztacala, UNAM. Tlalnepantla.

Montoya-Esquivel, A. 1998. Ethnomycology of Tlaxcala, Mexico. McIlvainea 13 (2): 612. 
Montoya A., Hernández-Totomoch A., Estrada-Torres A., Kong A. 2000. Recetas tradicionales para cocinar hongos silvestres. Fundación produce Tlaxcala. Folleto técnico $\mathrm{N}^{\circ} 20$.

Montoya A., Estrada-Torres A., Kong A y Juárez-Sánchez L. 2001. Commercialization of wild edible mushrooms in three markets of Tlaxcala, Mexico. Mycological Palisade international 13(1):31-40.

Montoya A, Hernández-Totomoch O, Estrada Torres A, Kong A. 2003. Traditional knowledge about mushrooms in a nahua community in the state of Tlaxcala, Mexico. Micología 95(5), pp 1003-131.

Montoya A., Kong A., Estrada-Torres A., Cifuentes J. Caballero J. 2004. Useful wild Fungíi of La Malinche National Park, México. Fungal Diversity 17: 115-143.

Montoya A., Estrada-Torres A., Kong A., Mapes-Sánchez C. 2004. Conocimiento Tradicional de los hongos en San Francisco, Temezontla, Tlaxcala, México, En Monroy. Aportes Etnobiológicos de la Red Regional de Recursos Bióticos. ISBN 968-878-160-6. ED UAEMor, México, pp. 166-214- UNAM. Instituto de investigaciones sociales. 88 p.

Montoya A. 2005. Aprovechamiento de los hongos silvestres comestibles en el Volcán la Malinche, Tlaxcala, Doctorado en ciencias. Facultad en ciencias. UNAM. México. D.F. $154 \mathrm{p}$.

Montoya A., Cortes, E Sánchez., Kong A., Torres-García. 2009. Conocimiento y tradicional de los hongos silvestres Altamira de Guadalupe Huamantla, Tlaxcala. En: Matlalcuéyetl: visiones plurales sobre cultura, ambiente y desarrollo. El colegio de Tlaxcala, México. Mesoamerican Research Foundation.

Montoya A. 1997. Etnomicología en la comunidad de San Francisco Temezontla, Estado de Tlaxcala. E.N.E.P., UNAM. Tlalnepantla.

Reyes-López R C. 2007. Clasificación tradicional de los hongos silvestres en San Isidro Buensuceso. Tesis de maestría. Universidad Autónoma de Yucatán.

Torres-García E A. 2007. Estudio ecológico de los hongos silvestres en la cañada grande, Parque Nacional la Malinche, México. Tesis de licenciatura. Universidad Nacional Autónoma de México (UNAM). Facultad de Ciencias Biológicas.

Vázquez N B. 2012. Conocimiento tradicional de los hongos en San Mateo Huexoyucan, Tlaxcala. Universidad Autónoma de Tlaxcala. Licenciatura en Biología. Facultad de Agrobiología. 
Zenteno P I. 2007. Conocimiento tradicional de hongos silvestres comestibles en Nanacamilpa, Tlaxcala. Tesis de licenciatura. Facultad de Filosofía y Letras. Universidad Nacional Autónoma de México. D.F.

Montoya A., Hernández N., Mapes, Kong A., Estrada-Torres A. 2008. The collection and sale of wild mushrooms in a community of Tlaxcala, Mexico Economic Botany 63(3): 413424.

Ramírez-Terrazo A. 2017. Importancia cultural de los hongos no comestibles en dos comunidades de las Faldas del Volcán la Malinche, Tlaxcala. Tesis de Maestría en Ciencias Biológicas (Sistemática), Instituto De Biología, Universidad Nacional Autónoma De México. 